\title{
DSP enabled Optical Detection Techniques for PON (Invited)
}

\author{
A. Teixeira, Member, IEEE, D. Lavery, Member, IEEE, E. Ciaramella, Senior Member, IEEE, \\ L. Schmalen, Senior Member, IEEE, N. Iiyama, Member, IEEE, R. Ferreira, Member, IEEE, S. \\ Randel, Member, IEEE
}

\begin{abstract}
Some of the different aspects of the DSP enabled receivers in access networks will be addressed in this work. A revision of the main topics which may impact DSP design will be discussed through application examples. Modulation formats, KK receivers and coherent receivers are analyzed at light of the DSP enabled receivers' impact. Also, relevant are FEC and burst mode which are debated at the light of PON requirements. Simplified analog coherent receivers are presented as a use case to serve as simplification path for non-DSP high performance receivers. On the other end, aiming at discussing current PON limits, an ultra dense wavelength division multiplexing (UDWDM) use case is also presented where several advanced topics, like pulse shaping, high density and simplified DSP operations are considered.
\end{abstract}

Index Terms - DSP, PON, DMT, KK, FEC, coherent, quasi coherent, UDWDM, Burst Mode.

\section{INTRODUCTION}

$\mathrm{T}$ HIS paper addresses the digital signal processing (DSP) enabled optical detection techniques for PON. It is covering a wide range of related topics which essentially aim at identifying the main paths towards the usage of DSP in PON networks receiver side.

With the evolution of traffic and users, data volume is already huge and growing continuously. A key point when considering the system strategy for future optical access networks is cost effectiveness, both in terms of CapEx (capital expenditure), including equipment, and OpEx (operational expenditure) [1]. This comes with the need for simple and costeffective implementations. Aiming at lower cost, all PON technologies, except NGPON2 or G.989, recurred to significantly loose wavelength bands for simpler band coupling/separation, intensity modulation due to its inherent generation/processing simplicity, O-band upstream for lower ONT laser requirements and dispersion impairments and burst mode Time Division Multiplexing (TDM) in order to share the bandwidth more flexibly.

The paper was submitted on the $23^{\text {rd }}$ june 2019 .

A. Teixeira is with Instituto de Telecomunicações and DETI/Universidade de Aveiro, 3810-193 Aveiro, Portugal (teixeira@ua.pt)

D. Lavery is with university college of London (UCL), Gower St, Bloomsbury, London WC1E 6BT, UK (d.lavery@ucl.ac.uk)

E. Ciaramella is with Scuola Superiore Sant'Anna, Pisa, 56124, Italy (e.ciaramella@sssup.it)
These trends have been around in the network and respective standards for the last decades in PON. NGPON2 was designed to be compatible with a designated WDM band and itself recurring to a combination of WDM and TDM in the $\mathrm{C}$ and $\mathrm{L}$ band, allowing backwards and future compatibility.

The pressure on cost and the need for wavelength usage evolution opened into PON space to many advanced techniques, such as advanced modulation formats by increasing the bit rate without increasing the electronics bandwidth; burst processing in complex contexts allowing more users; FEC related evolutions allowing wider choice at component level or higher tolerance to impairments; simplified coherent detection schemes allowing filter-less and higher sensitivity devices [2][3] and advanced WDM solutions allowing higher density and usage of spectrum.

Whilst coherent detection can allow for higher power budget and UDWDM operation, the use of expensive components and complex DSP can limit their deployment in PON [4]. Therefore, various solutions were proposed and demonstrated that achieve coherent detection by means of simplified processing, often analog processing [5]-[12]. The key benefit of this approach relies on the simplified operation, lower power consumption and higher cost effectiveness (analog processing is often combined with common COTS components). The approach is compatible with WDM operation, and its cost benefits have been assessed [11]. However, whilst the operation till $10 \mathrm{Gbit} / \mathrm{s}$ is well-proven, higher speed values are still an open point. Indeed, it is not demonstrated that the analog processing can support real time operation at very high speed (e.g. $50 \mathrm{Gbit} / \mathrm{s}$ ), mainly because of the limited speed of present analog electronic devices.

Parallelly, optical access networks (OANs), open some space for potential usage of coherent systems to better exploit the available wavelength spectrum and extend the capacity of the current PONs [17]. Combined with DSP, coherent enables highly flexible networks using software-defined transceivers, which will benefit future PON systems with dynamic reconfigurability, enabling to adapt the channel conditions or bandwidth requirements by working with a variety of

L. Schmalen is with Karlsruhe Institute of Technology (KIT), Kreuzstr. 11, 76133 Karlsruhe, Germany (Laurent.Schmalen@ kit.edu ),

N.Iiyama is with NTT Corporation, Yokosuka, 2390847 Japan (Noriko.iiyama.sk@hco.ntt.co.jp).

R. Ferreira is with PICadvanced, 3830-352 Ilhavo, Portugal. (Ricardo.ferreira@picadvanced.com )

S. Randel is with KIT (Karlsruhe Institute of Technology), Engesserstr. 5, 76131 Karlsruhe, Germany, (Sebastian.randel@kit.edu ) 
modulation formats, different symbol rates or digital equalizers. For UDWDM-PON for instance this may be a key aspect due to different link power budgets of the different users or due to the nature of dense wavelength allocation that may result in different channel performances.

This work will continue with a general overview on the modulation formats and receiver concepts for PON, where discussions will allow observing its implications on the DSP based receivers. In what follows, FEC is going to be addressed since it enables cheaper PON systems and higher number of clients hanging on the network by increasing the tolerance to higher optical losses and distortions. Besides FEC, the burst mode is one of the approaches to allow sharing of the physical media and results in extra requirements to DSP based receiver. A simplified coherent approach is also addressed to allow exploiting the possibilities of analog DSP simplified receivers. A higher complexity DSP based system, UDWDM, is described in terms of challenges and some of the approaches for simplification are discussed. Finally, conclusions are drawn.

\section{ADVANCED MODULATION FoRMATS AND RECEIVER CONCEPTS FOR PON}

Despite all generations of PON to date being exclusively based on on-off keying using intensity modulation and direct detection (IM-DD), there is a renewed interest in introducing advanced modulation formats and, possibly, coherent detection. When using advanced modulation formats, there will always be a trade-off between spectral efficiency, receiver sensitivity, and DSP complexity. While on-off keyed systems can generally operate without DSP, the introduction of, for example, 4-ary pulse amplitude modulation (PAM) increases the signal-to-noise ratio requirements and thus decreases the tolerance to the penalty introduced by chromatic dispersion. With higher-order modulation formats, maintaining the required link budget will require higher transmit powers, which will ultimately be limited by fiber nonlinearities and eventually by eye-safety regulations.

Despite the lack of phase information in IM-DD signals, chromatic dispersion penalties can be partially mitigated through the use of a digital finite impulse response (FIR) filter for feed-forward equalization (FFE). There is, therefore, a tripartite optimization process when determining DSP requirements for PAM systems. (1) Determine the system length, (2) set the modulation format that meets the transmission system's SNR, and (3) determine the symbol rate requirements for that format (e.g., PAM-4 requires half the symbol rate of on-off keying (OOK)), to determine the FFE requirements. By iterating over this process, it is possible to design a signal which is tuned to the requirements of the transmission system. Ultimately, this process becomes a tradeoff between the high order modulation, and the DSP performance. Although the exact DSP requirements vary depending on the link configuration, the typical range for the number of FIR filter taps required is $15-25$.
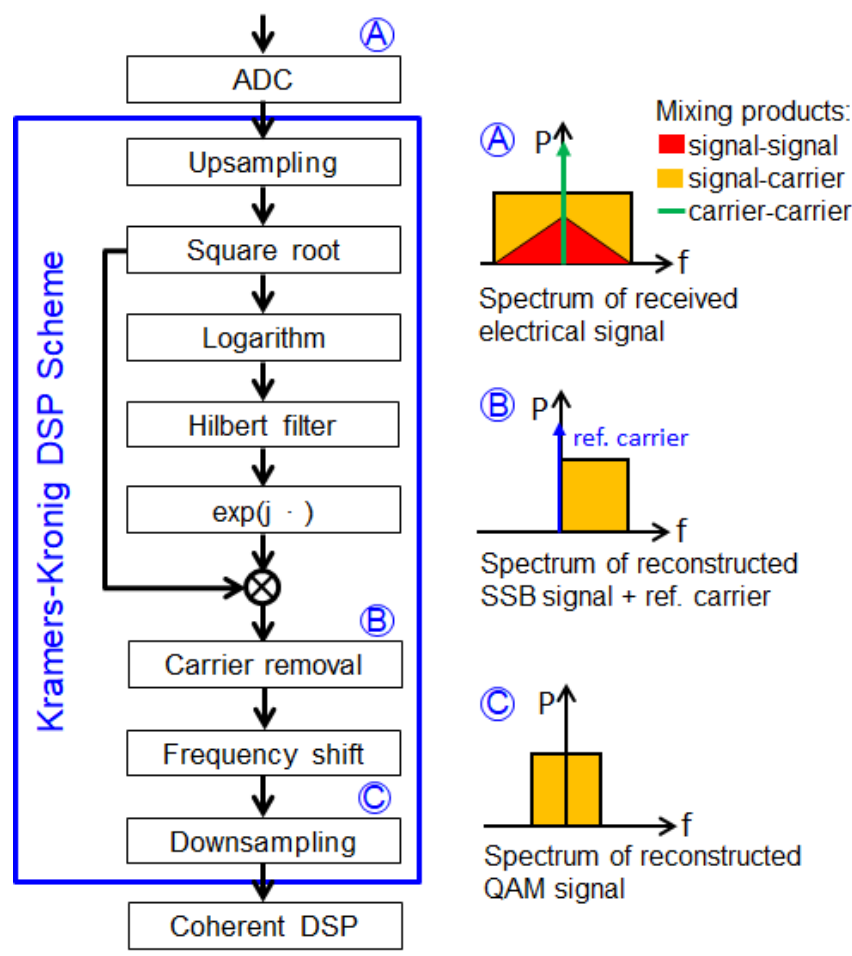

Spectrum of reconstructed SSB signal + ref. carrier

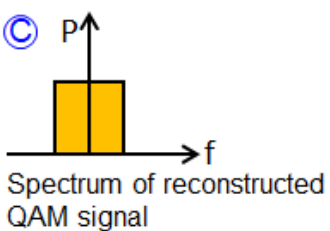

Figure 1 - Overview of the KK receiver DSP chain and schematic representations of the signal spectra at the indicated positions.

However, because chromatic dispersion is a function of the signal bandwidth, it is possible to reduce the requirements of the DSP by limiting the spectral width of the signal. Optical duobinary (ODB), for example, has been investigated for this reason. ODB introduces correlation between consecutive signals; the autocorrelation function of this signal is designed to reduce the spectral width, without requiring the relatively high DSP complexity of pulse shaping filters. However, this approach is ultimately limited by chromatic dispersion, as for all IM-DD systems. Therefore, the following subsections describe alternatives to IM-DD and ODB that will also help to circumvent this restriction.

\section{A. Kramers Kronig (KK) Receivers}

Two further signaling options for direct detection systems are single-sideband (SSB) modulation and discrete multitone (DMT). SSB uses an external modulator to produce a frequency upconverted signal which is transmitted alongside a carrier tone. The signal is received using a single photodetector, but retains the signal's phase information due to the self-coherent mixing between the signal and the co-propagating carrier tone, which enables the use of advanced modulation formats such as 16-ary quadrature amplitude modulation (QAM). In order to produce an SSB signal with a reasonable optoelectronic bandwidth requirement, the signal must be spectrally shaped (generally with a root-raised cosine filter, with a typical roll-off of 0.1). For improved performance, a similar filter response must be applied to the received signal. Both of these techniques introduce DSP complexity, although there is a trade-off between filter requirements and spectral width. Due to the selfcoherent detection, a chromatic dispersion compensation filter 
can be applied using the same FIR filter design as is used in coherent transmission systems [18][30]. However, the overall performance of the system is limited by the noise and the power of the pilot tone, and also signal-signal beat interference (SSBI) due to the use of direct detection. SSBI can be almost entirely compensated using Kramers-Kronig (KK) receiver DSP [19][30]. The DSP chain for KK is shown in Figure 1, and includes a square-root function, a logarithm, and a Hilbert filter. Additionally, due to the nonlinear operations in the DSP, the DSP must be applied to an oversampled signal, with a typical oversampling of 4 samples/symbol. By contrast, DMT deals with chromatic dispersion by transmitting narrow-band sub-carriers, each of which can be bit-loaded to produce a signal which encodes an information rate matched to the frequency-dependent SNR of the system [20]. The DSP requirements for this are an IFFT/FFT pair, which is required to apply frequency-dependent modulation as well as single-tap per-subcarrier equalization. If a carrier is transmitted with the DMT modulation (as is typical), and the signal is prefiltered to be single-sideband, then KK can also be used to linearize the receiver, enabling digital dispersion compensation.

\section{B. Coherent Receiver}

For PONs with data rates of $100 \mathrm{Gbit} / \mathrm{s}$ or more, phase- and amplitude modulated signals, in combination with high sensitivity coherent reception, might become the only viable option. In contrast to direct detection, coherent receivers beat the received optical field with an optical reference carrier, commonly referred to as the local oscillator (LO). A key advantage of the LO, versus self-coherent transmission, is that it permits balanced detection, which inherently removes the SSBI terms that occur with single-ended detection.

In long-haul systems, a polarization-diverse digital coherent receiver is usually employed to ensure polarization matching between the signal and LO, and to allow for polarizationdivision multiplexing. For PONs, this presents a technical challenge due to the complexity of the opto-electronic coherent receiver circuitry. Typical polarization-diverse coherent receivers consist of two polarization beam splitters, two 90degree optical hybrids, as well as 4 balanced photodiodes followed by transimpedance amplifiers and four analog-todigital converters (ADCs). In recent years, significant progress has been made in reducing the footprint, the power consumption and the cost electronic-photonic integrated circuits [31][32], however, the especially low complexity requirements of an ONU have yet to be met by commercial receivers.

Simplified coherent receivers and Ultra dense WDM (UDWDM), which could be used for PONs, are discussed elsewhere in this manuscript, and will not be discussed in detail here. However, it suffices to say that it is possible to avoid the requirement for polarization diversity (thus halving the receiver complexity), provided the signal is appropriately precoded [25][26]. Additionally, a heterodyne configuration can be used (analogous to the self-coherent scheme detailed previously), to reduce the opto-electronic receiver complexity to a single balanced photodiode at the expense of higher bandwidth and lower spectral efficiency. Note that if a single-ended photodiode is used in this configuration, $\mathrm{KK}$ reception is once again required to eliminate the SSBI.

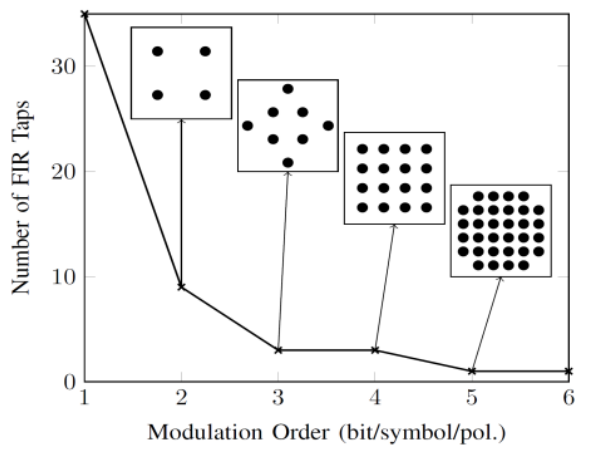

Figure 2 - Required number of chromatic dispersion compensation filter taps for different coherently detected modulation formats (assuming $25 \mathrm{~Gb} / \mathrm{s} /$ polarization C-band signals transmitted over 100 km). Inset figures are 4-, (star) 8-, 16-, and 32-QAM.

Regardless of the configuration, the coherent receiver outperforms the direct detection receiver because the power of the reference carrier is not depleted in transmission. This provides the flexibility to use high LO-signal power ratios, thus achieving linear detection but with low signal powers. This unique combination enables not only an increased receiver sensitivity but also electronic post-processing which can greatly improve the overall system performance. This leads to the question; how can we use simplified coherent receivers to upgrade IM-DD systems, and what are the DSP implications?

The simplest upgrade to IM-DD systems, namely on-off keyed coherent systems, do not inherently require DSP, and yet can offer sensitivity gains versus their IM-DD counterparts [21]. However, this is not making the best use of the digital coherent receivers, as they offer the flexibility of advanced modulation [22][23], and can also exploit the linear detection profile for perfect chromatic dispersion compensation.

While for IM-DD there is a relationship between the chromatic dispersion tolerance and the modulation format, for digital coherent receivers no such relationship exists. Instead, the required number of FIR filter taps depends only on the accumulated dispersion within the signal [18]. Figure 2 shows the required number of chromatic dispersion filter taps for a reasonable PON data rate of $25 \mathrm{~Gb} / \mathrm{s}$, and C-band transmission over a distance of $100 \mathrm{~km}$; commensurate with a long-reach PON. Although PONs are conventionally operated in both the C- and L-bands, a coherent receiver's insensitivity to dispersion means that the C-band is preferred due to the lower fiber attenuation. As the order of modulation is increased, the required symbol rate correspondingly decreases. With an 8 -ary 
modulation, only 3 filter taps are required for chromatic dispersion compensation (note that, due to the quantization of the number of chromatic dispersion filter taps, 3 taps are also sufficient for 16-ary modulation). Such levels of chromatic dispersion are negligible and would not necessarily need to be compensated using a separate FIR filter; instead being compensated as part of an adaptive equalizer. Indeed, even for 4QAM, fewer than 10 filter taps are required, meaning that 4QAM offers a good tradeoff between DSP complexity and the increased SNR requirements of higher order QAM formats.

Indeed, the DSP complexity of digital coherent receivers (in the context of a PON) comes from the adaptive equalization and, to a certain extent, the carrier phase estimation. Dual polarization modulation schemes, which are used in long haul transmission systems, require an adaptive multiple-input multiple-output (MIMO) filter to track the state of polarization of the received signal. These filters are highly complex, and contribute significantly to the power consumption of the DSP chain [24]. Ultimately, if per-channel data rates in PON are to scale significantly, dual polarization transmission will be required, but scaling trends in CMOS will likely be ready to support this when required [29]. Finally, it is worth noting that the DSP chain for coherent receivers can be further simplified by using differential decoding with quadrature phase shift keying (QPSK). This introduces a power penalty (which differs depending on implementation [27]), but relaxes laser linewidth and carrier phase estimation requirements. The low DSP complexity of this combined approach has been recently demonstrated by using realtime GPU processing for the coherent receiver DSP chain Error! Reference source not found.. Although differential decoding is not scalable to higher order modulation formats, it does provide a means to implement coherent receivers suitable for UDWDM systems.

\section{FORWARD ERROR CORRECTION (FEC) IN PONS}

Forward Error Correction (FEC) is an indispensable technology in many communication system. The application of FEC enables significant gains in tolerable receiver signal-tonoise ratio (which is especially important if we operate in a power-limited regime) at the expense of an increase in gross data rate to transmit parity bits and processing cost. Early optical communication systems operated often without FEC, but even a very simple FEC can lead to significant SNR gains and hence the use of FEC is strongly encouraged. In 1988, very basic FEC schemes were proposed for optical communications [41], showing performance improvements with respect to the required received power and the observed residual error floor, but also with respect to chromatic dispersion tolerance and resilience to laser mode hopping.

Today, only few systems that are optimized for ultra-low complexity operate without FEC. As FEC is a ubiquitous technology, there exists a plethora of different schemes optimized for a variety of applications. Figure 3 qualitatively illustrates the performance of different FEC schemes with respect to an uncoded transmission. The theoretical limit is the performance that can be obtained with an ideal FEC scheme and infinite resources. Today's modern strong FEC schemes (e.g., a typical staircase code [42]) operate very close (within $0.5 \mathrm{~dB}$ ) to the theoretical limits (see Figure 3) and offer 3-6 dB additional coding gains compared to older, weak FEC schemes like, e.g., Reed-Solomon (RS) codes often employed today in PONs (see below). For the non-expert, it may be difficult to get an idea which schemes could be beneficial and helpful in their use. Even in the field of optical communications, it is hard to give a clear indication of a single FEC scheme that suits all applications, as the requirements for the different applications can differ quite drastically: In long-haul terrestrial and submarine cables, usually a large net coding gain (NCG) is of utmost importance. Hence, significantly more hardware complexity (e.g., receiver chip area and electrical power) is available in contrast to PONs, which are installed at the enduser premises. In short-reach optical communication systems, e.g., for data-center interconnects, staircase codes seem to become state-of-the-art FEC technique [42]. However, staircase codes suffer from a non-negligible latency due to the windowed decoding of multiple blocks of several 100kbit. Hence, staircase codes are well-suited for applications that act as data pipes with constant traffic, but are less suited for systems with volatile traffic and transmission of short packets (as is the case for PON, in particular for uplink from ONU to OLT).

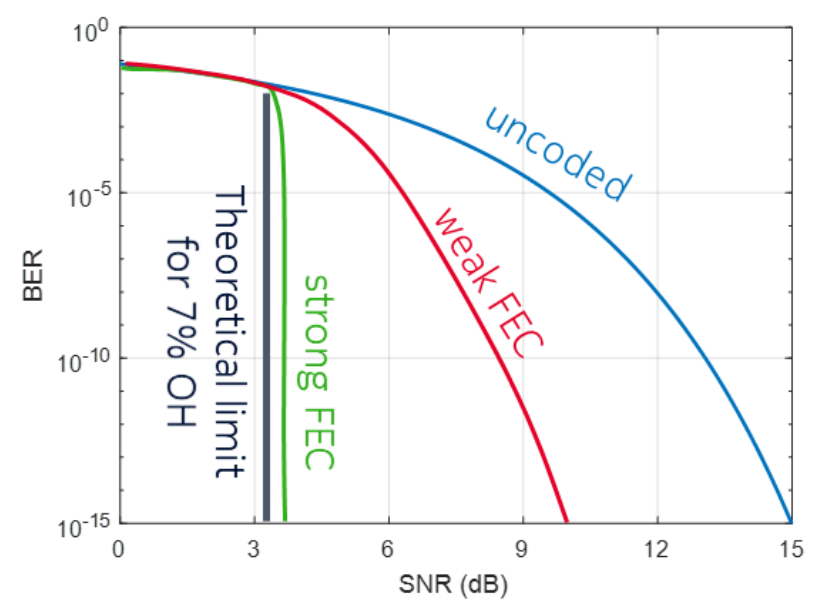

Figure 3 - Performance of FEC schemes with $7 \%$ overhead $(\mathrm{OH})$ (Rate $\approx 0.93$ ). This figure has been obtained using binary modulation (e.g., 2-PAM) and hard-decision decoding, i.e., binary FEC decoder input.

The application of modern FEC in PONs poses various challenges. First, PONs are, by design, strongly asymmetrical systems: The ONU, placed at the end-user premises, can only support a certain amount of complexity (both hardware and 
computational), while the OLT can spend significantly more resources in processing the signals. For these reasons, very simple FEC schemes were employed in PON systems up to today. Most of these simple schemes are based on RS codes introduced in 1960 by Irving Reed and Gustave Solomon. RS codes are nonbinary cyclic error correcting codes that are able to correct and/or detect a predetermined number of erroneous nonbinary symbols, consisting of groups of bits. The corresponding algebraic decoding algorithm was proposed later by Berlekamp and Massey. A widely used code is the Reed-Solomon code RS $(255,239)$ consisting of 255 nonbinary symbols with each symbol composed of 8 bits. This code can correct up to eight symbol errors (or a single burst error of up to 57 bits) with $6.7 \%$ overhead and achieves a net coding gain (NCG) of $6.2 \mathrm{~dB}$ in signal-to-noise ratio (SNR) at a post-FEC BER of $10^{-15}$. This means that we can tolerate an effective net SNR, considering the extra bandwidth required for the coding overhead, that is 4 times lower than without coding. This ReedSolomon code is the first that found widespread use in fiberoptic communication systems and optical access systems [43].

Today, there is a quest for higher coding gains without adding extra latency. Hence, new coding schemes are required. In a recent standardization effort, low-density parity-check (LDPC) codes have been adopted for PON systems, in particular for the 802.3ca standard [44]. LDPC codes have many advantages that make them well suited for PON systems. First, due to their popularity, very efficient hardware decoder architectures have been designed. Secondly, they achieve high net coding gains with moderately large block lengths and hence low latencies, making them well suited for PONs. Finally, LDPC codes are future-proof due to their universality property: today's PON systems mostly do not use analog-to-digital conversion and the decoder needs to cope with bits. In the future, when ADCs become widespread in PONs, the same code and the same decoder can be used to carry out softdecision decoding (only a small preprocessing unit needs to be exchanged) giving 1-2 dB extra NCG.

Like many other FEC schemes, the rate of LDPC codes can be adapted as well by the techniques of shortening and puncturing, enabling the use of information sequences of varying length. In shortening, some of the information bits are set to zero before encoding and not transmitted. The decoder is aware of this fact and fills the missing bits with the zero value. With this technique, the length of information sequences can be adjusted (e.g., to accommodate short blocks like "acknowledge" packets) while being able to reuse the same decoder hardware. On the other hand, puncturing allows to reduce the overhead of the FEC scheme by not transmitting some parity bits and flagging them as "unknown" at the decoder. The receiver tries to recover these missing parities besides the payload information, at the expense of a performance loss. Furthermore, LDPC codes can cope relatively well with burst errors. The burst error correction capabilities can be improved further by adding interleaving.

On the transmit side, the encoding of LDPC codes is usually more complex than the encoding of more classical codes like RS codes (which can be encoded using shift registers). However, the complexity of LDPC encoding circuits can be managed by employing special code structures that target simple encoder circuits based on accumulators and barrel shifters without significantly sacrificing decoding performance.

The vision of having a FEC scheme that can be used in the next decades is probably one of the major drivers to adopt LDPC codes in future PON standards.

\section{DSP BASED BURST MODE OPERATION}

PON systems can be based on TDM scheme due to its potential cost effectiveness and wide use in various PON standards, the upstream signals are in burst mode and the receiver at the OLT side needs to cover burst mode operations. Since time and wavelength division multiplexing (TWDM)-based PON systems also have the same requirements due to their TDM component, most PON standards have to have an OLT receiver to deal with the burst signals.

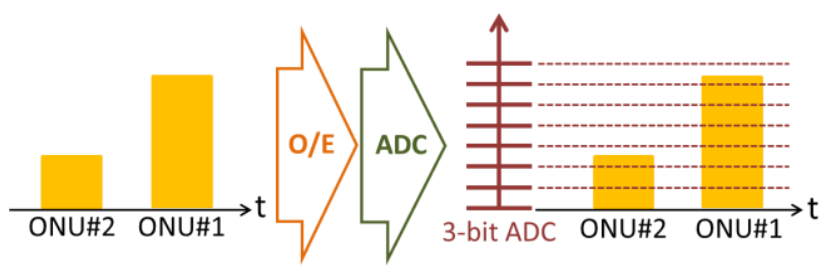

Figure 4 - Amplitude inequality impact in ADC resolution

Although receiving burst signals is not an easy task even for analog intensity modulation - direct detection (IM-DD) PON systems without DSP such as 10G-EPON - DSP based systems have specific difficulties when dealing with the burst signals due to their characteristics; the frames are discontinuous and their amplitudes and impairments vary for each burst frame because the frames are transmitted via different paths from different ONU transmitters.

Regarding the amplitude inequality, the required dynamic range is as much as $20 \mathrm{~dB}$ in most PON standards. This prevents making full use of vertical resolution of ADCs, which are necessary for DSP-based systems. Figure 4 is an example to simply explain the problem. When two burst signals that have small amplitude and large amplitude are digitized via, e.g. a 3-bit ADC that has 8 levels, the input signals and the ADC are adjusted so that the amplitude of the larger signals, the signal from ONU \#1 in Figure 4, becomes an optimal value of the ADC to digitize the whole signal. Then, the smaller signal 
from ONU \#2 uses only 4-level that corresponds to 2-bit even though the ADC has 3-bit resolution. This means that the vertical ADC resolution cannot be fully utilized. To avoid that, the frames need to be leveled before the ADCs.

A number of approaches that level the burst signals have been reported thus far. In a PON system such as 10G-EPON, which transmits OOK signals without DSP, electrical amplifiers such as a transimpedance amplifier (TIA) and a limiting amplifier (LA) designed to receive the burst signals are employed to level the burst frames. However, for a DSP-based PON system that needs better amplitude resolution than analog OOK in order to deal with waveform information, those electrical amplifiers cannot directly be applied because amplitude information beyond OOK is lost.

For that requirement, although burst-mode LA which is designed only for OOK signals inherently cannot be used, burst-mode TIA can be used if it is developed to have sufficient linear response region. A $50 \mathrm{~Gb} / \mathrm{s}$ PAM-4 burst-mode upstream transmission with direct detection that employs a linear burstmode TIA is demonstrated by Coudyzer et al. [45]. The system achieves an input dynamic range of $10.9 \mathrm{~dB}$.

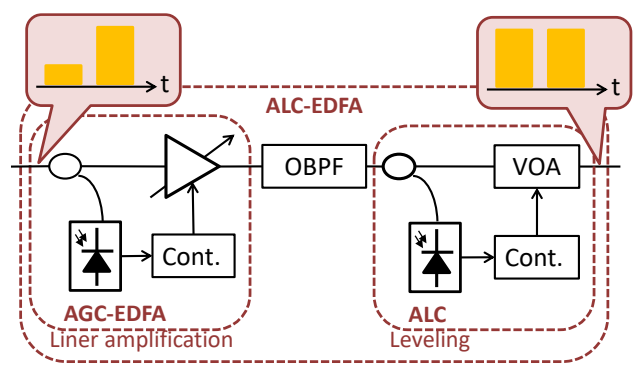

Figure 5 - ALC-EDFA in ref. [46]

Another solution is using optical amplifiers to level the burst signal amplitudes. A $20 \mathrm{~Gb} / \mathrm{s}$ QPSK burst mode system with the automatic level control erbium-doped fiber amplifier (ALC-EDFA) shown in Figure 5 followed by a coherent receiver that achieves receiver sensitivity of $-44.7 \mathrm{dBm}$ and dynamic range of $21.7 \mathrm{~dB}$ after $40 \mathrm{~km}$ SMF transmission is reported [46]. This ALC-EDFA consists of three parts: a burstmode automatic gain controlled (AGC) -EDFA, an optical band-pass filter (OBPF) and a burst-mode ALC circuit that is a variable optical attenuator (VOA) controlled by a feed-forward circuit. The AGC-EDFA amplifies signals linearly to satisfy the input power range allowed by the following ALC circuit and the ALC circuit levels the linearly amplified signals so that linear TIAs are not saturated.

When a DSP-based PON system employs coherent detection, i.e., digital coherent technology, as the above one, the LO can be a leveling component by controlling its input power to a $2 \times 4$ optical $90^{\circ}$ hybrid [47]. Figure 6 shows a coherent receiver based on the power-controlled LO. LO power, $P_{L O}$, is attenuated by a controlled VOA so that $P_{S} P_{L O}$, which is the product of the signal input power $P_{S}$ and $P_{L O}$, is kept to a constant value. $P_{S}$ can be obtained either by detecting the burst signal input before the $90^{\circ}$ hybrid or by estimating it using information sent from the dynamic bandwidth allocation (DBA) scheduler and the operational support system (OSS). In the latter case, OLT arrival time is also required. A $20-\mathrm{Gb} / \mathrm{s}$ orthogonal frequency division multiplexing-PON (OFDMPON) with coherent detection based on a power-controlled LO that achieves a receiver dynamic range of $25 \mathrm{~dB}$ and a system power budget of $29 \mathrm{~dB}$ is reported [47].

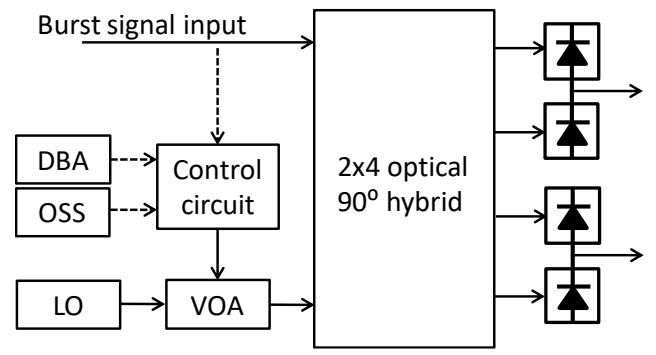

Figure 6 - LO power control based coherent detection in ref. [47]

In response to high-speed and long-reach demands, nearfuture PON standards will most likely require over $20-\mathrm{dB}$ dynamic range at a rate of $25 \mathrm{~Gb} / \mathrm{s}$ or beyond. If technical issues and cost are taken into consideration, it will be hard to meet the above mentioned requirements with a single component even using all above-cited approaches (linear burst TIA, ALC optical amplifier, and power-controlled LO) because of its inherent merits and demerits; In general, electrical approaches can be realized with lower cost while optical approaches can achieve higher sensitivities and dynamic ranges. Combining some of these approaches also could be a practical solution.

As for other burst signal characteristics, the discontinuity and the different distortion characteristics for each burst frame affect the convergence time of adaptive equalization filters in the DSP. With continuous systems such as core \& metro networks, the adaptive filters can take the time to make their coefficients converge and the payload can keep using the coefficients once they converge. On the other hand, with burst systems, since the coefficients differ for different distortion characteristics, a burst frame transmitted from an ONU cannot use the coefficients for the previous burst frame transmitted from another $\mathrm{ONU}$ and the filter needs some time to make the coefficients converge at the beginning of every frame (Figure 7). Especially when a DBA timeslot is filled with frames, an allowed payload length is limited by the preamble length of the frame. That means that a longer preamble length leads to a shorter payload length and deterioration of the transmission efficiency. Therefore, the coefficients are required to converge as rapidly as possible so that the transmission efficiency is not deteriorated. 
A solution shown in Figure 8 which uses constant modulus algorithm (CMA) as an algorithm of the adaptive filter is calculation of the coefficients in the discovery process and handover [46]. In the discovery process, the coefficients converge within the preamble of the register request that is set relatively long so that the convergence is completed. The converged coefficients are stored in a memory and linked with a logical link identifier (LLID) that is unique for each ONU.

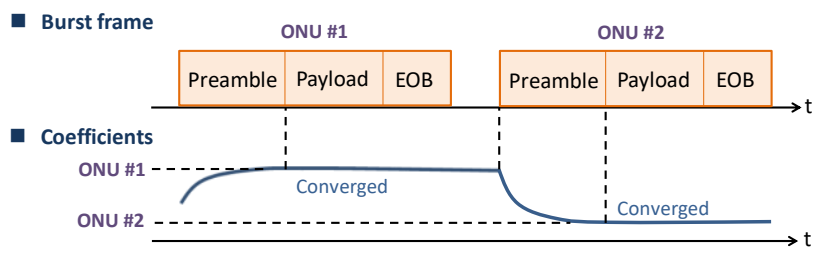

Figure 7 - Coefficient behaviors of adaptive filter in burst mode.

This process is repeated for all ONUs. In the DBA process after the discovery process, LLID of a frame incoming to the OLT is identified from the gate frame information before the burst frame arrives at the OLT. The coefficients linked with the LLID are then read from the memory and set as the CMA default values. The frame can be processed with the converged coefficients from the beginning of the frame. The memory is updated by the coefficients at the end of each frame. In this process, not much time is required for the coefficients to converge at the beginning of each burst frame. In consequence, no long preamble is required in the DBA process and transmission efficiency is not deteriorated. A successful detection of a $20 \mathrm{Gbit} / \mathrm{s}$ QPSK burst signals with a short preamble length of $1.3 \mu$ s is reported [46].

Note that the longest duration that an ONU is not active is less than some milliseconds in a general case such as EPON Multi-Point Control Protocol (MPCP) that cuts the connection with the OLT if the ONU is not active for more than the period regulated by "MPCP timeout." Signal characteristics such as state of polarization (SOP) do not change dramatically in such a short period and the stored coefficients can be used without concern for that. If the connection is cut due to the MPCP timeout, the discovery process is done again when the ONU is reconnected to the OLT. In that case, the coefficients are also calculated in the discovery process again. TDM is basically a cost effective choice compared to other multiplexing methods such as WDM in terms of the maximum capacity that a subscriber can utilize thanks to its statistical multiplexing effects. The approaches introduced in this section could undermine this cost advantage because of the additional devices and DSP part while WDM also needs some optical components that TDM does not require. The best choice always depends on the application requirements. Before standardizations or developments, the total system costs including maintenance and operation costs have to be well discussed.

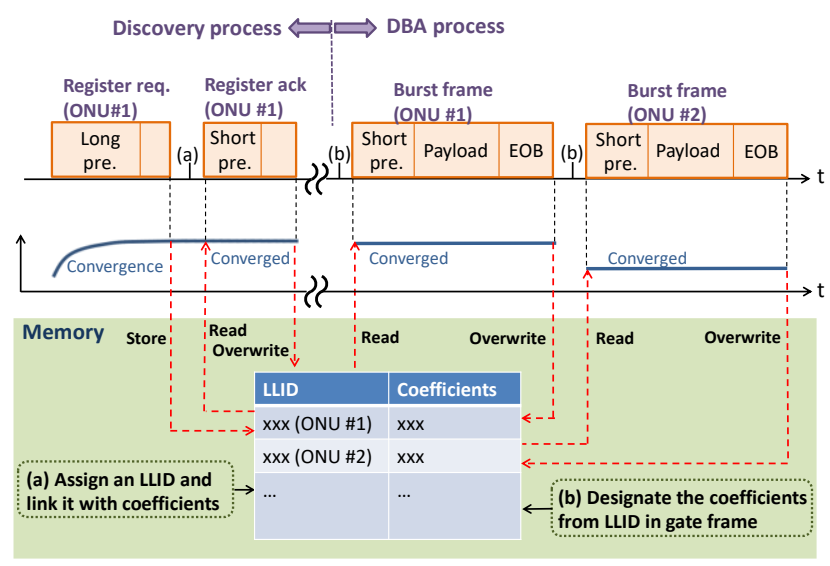

Figure 8 - Calculation of coefficients in discovery process [46].

\section{DSP-FREE COHERENT DETECTION SCHEMES}

In various PON applications, using DSP can present issues, due to latency, power-consumption and even cost. It is therefore important that new solutions based on simplified schemes were proposed and demonstrated. Simplification comes with a reduction of functionalities, which, however, can be willingly accepted by operators who would afford much lower costs [11]. These concepts are summarized in Table I.

In coherent systems, three main functionalities are usually performed by DSP, which are carrier frequency recovery, polarization recovery and detection of in-phase and inquadrature multi-level signals. In most of the proposed simplified solutions, Intensity modulation (IM) or duobinary (DB) is used, both of which require no specific carrier frequency recovery: in that case, the LO frequency can be set by means of current temperature controllers with good enough accuracy, so that carrier recovery is not required.

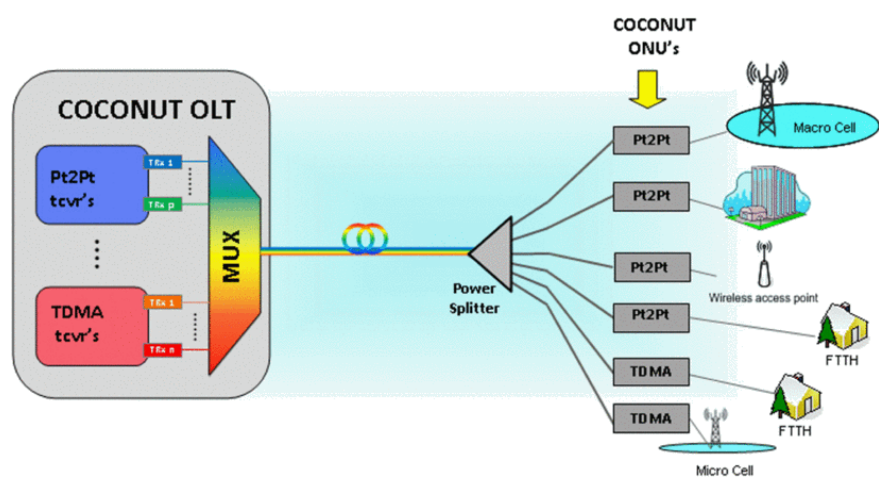

Figure 9 -Scenario of COCONUT network. Pt2Pt: point-to-point; TDMA-Time Division Multiple Access; FTTH-Fiber-To-The-Home; tcvr-transciever.

Similarly, polarization recovery is not needed once you choose to have single-polarization signals. In that case, clearly, you then should use a polarization-independent detection 
scheme. Finally, IQ detection may not be needed in PON, so that, using simpler modulation formats common RX electronics can be used (e.g., the CDR used for NRZ signals)

TABLE I-

COMPARING DSP FUNCTIONALITIES AND EQUIVALENT SOLUTIONS FOR DSPFREE IMPLEMENTATIONS

\begin{tabular}{cl}
\hline \hline $\begin{array}{c}\text { DSP FUNCTIONALITIES IN } \\
\text { METRO-LH }\end{array}$ & SIMPLIFIED SOLUTIONS FOR ACCESS \\
\hline Carrier frequency recovery & $\begin{array}{l}\text { Use IM, with relaxed limits of carrier- } \\
\text { LO stability }\end{array}$ \\
Polarization table recovery & No PolMux, Use Pol-Ind RX
\end{tabular}

Multi-level IQ signal detection

Simpler binary or multilevel schemes

In the area of simplified coherent detection, the first structured proposals came from the project COCONUT [4],[39]. The envisioned target was to achieve increased powerbudget in UDWDM-PON, by means of simplified coherent detection solutions. A particular effort was devoted to realize and prove simplified detection schemes for both NRZ and DPSK signals, removing the most expensive components both photonic and electronic. The reference network scenario, reported in Figure 9, included various applications, such as antenna remotization, backhauling for small and macro cells, connection for residential and business users. The presented scenario, clearly, can be considered as reference also for the other simplified coherent detection solutions.

A key requirement for simplified coherent realization was to remove DSP, exploiting analog processing. Since DSP takes care of several functionalities, a complete re-working was needed. In particular, for NRZ, a phase diversity scheme based on $3 \times 3$ coupler was selected [34], since this can realize coherent detection without careful control of the LO frequency, the solution removes the need for DSP carrier recovery and can rely on analog processing [35], [36]. As another relevant feature, the scheme was extended to achieve polarizationindependency without polarization-diversity by exploiting the 3 rd unused input of the $3 \times 3$ coupler [21], [8] as shown in Figure 10. Indeed, polarization diversity would double the receiver structure, which results into an excess cost of components. At same time, this solution removes the need for polarizationhandling at DSP level.

The experimental verification was attained by real-time processing at $1.25 \mathrm{Gbit} / \mathrm{s}$ (Gigabit Ethernet speed) and by realtime analog processing), it achieved polarization-independence with a very limited penalty compared to the manuallyoptimized polarization-sensitive solution, It requires intradyne operations, which might become advantageous when considering bidirectional operations [8]. This solution was successfully tested in a field trial over the metro network of Pisa city [39]. Similar implementation can be used on DPSK signals. DPSK allows in principle for higher sensitivity [12], but the price for this is that DPSK analog processing requires more accurate control of the LO.
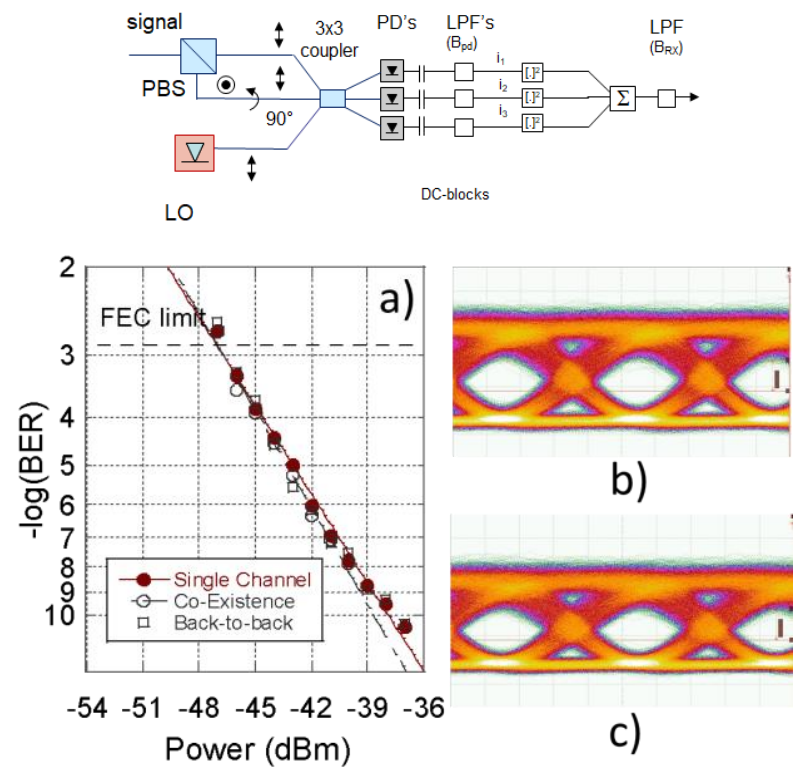

Figure 10 -Scheme of the PI-RX based on $3 \times 3$ coupler, and BER curves $a$ ) and eye diagrams $b, c)$ taken by the real time $R X$ implementation during the COCONUT field trial

Alternative concepts were recently presented with real time implementation and refinements starting from the polarizationindependent receiver introduced by Glance Error [33], for either IM [5] or DPSK signals [12]. Using IM, the RX was successfully implemented till $10 \mathrm{~Gb} / \mathrm{s}$, by using analog electronic processing. The IM format allows for simpler control of the detuning between signal and LO and can work in homodyne or intradyne regime. The related analog processing is also very simple, its main building block being a pair of envelope-detector circuits.

In addition, it was proven that the RX proposed in [5] also allows for VCSEL-based transmitters and LO: this feature makes it very promising for low-cost implementation.

To have the equivalent scheme working with DPSK, the receiver should work in the heterodyne regime [12]. Heterodyne can pose limitations due to higher requested bandwidth of optical and electronical devices; especially when moving to even higher bit rates, this feature can give dramatic limitations. As another side effect, the heterodyne approach is less spectrally efficient. It was demonstrated that PI-RX with IM can achieve ultra-dense spacing (see later), whilst this cannot clearly be attained by heterodyne. This can be partly compensated if the LO output is partly split and one part is used as transmitter source for the opposite transmission direction.

It should be outlined that all the above presented schemes are able to work with DML transmitters, which represents an additional bonus for PON networks (DML sources usually have lower cost and complexity). The different experimental 
implementations give similar sensitivity values.

TABLE II-

OBTAINED SENSITIVITIES FOR SIMPLIFIED DETECTION SCHEMES

\begin{tabular}{|c|l|l|l|}
\hline RX SCHEME & $\begin{array}{c}\text { MODULATION } \\
\text { FORMAT }\end{array}$ & \multicolumn{1}{|c|}{ SENS. (FEC LEVEL) } & REF \\
\hline PI 3x3 RX & IM & $\begin{array}{l}-48 \mathrm{dBm}(1.25 \mathrm{~Gb} / \mathrm{s}) \\
-40(10 \mathrm{~Gb} / \mathrm{s})\end{array}$ & $\begin{array}{l}{[8]} \\
{[39]}\end{array}$ \\
\hline PI Intradyne. & IM & $-35 \mathrm{dBm}(10 \mathrm{~Gb} / \mathrm{s})$ & {$[5]$} \\
\hline PI -3x3 Heter. & DPSK & $-37 \mathrm{dBm}(1.25 \mathrm{~Gb} / \mathrm{s})$ & {$[12]$} \\
\hline
\end{tabular}

Although DPSK might have in principle a much better sensitivity, yet presented results still do not show that, probably due to specific implementations. It is on the other side understood that the final choice among these implementations could be due to other issues (final cost, reliability, power consumption etc.) that, presently are difficult to estimate fairly for the three schemes. Table II presents the Sensitivities for simplified detection schemes.

Finally, in any choice for future large-scale implementations, a strategic issue would be the potential for simple transceiver schemes to work at 25 or even $50 \mathrm{Gbit} / \mathrm{s}$ rates, either using PAM-4, DQPSK, duobinary or simple NRZ format. This is still an open issue: in principle, all DSP-free schemes have no intrinsic speed limitations, all of them can indeed be used for higher rates (either binary or multilevel). As example, $25 \mathrm{Gbit} / \mathrm{s}$ with duobinary and PI-RX was recently obtained [40].

However, all of these DSP-free schemes critically rely on analog electronic blocks. Thus, we expect that the availability (and cost) of the required commercial devices might represent an issue for any of these schemes to work at higher rates.

\section{DSP (ULTRA DENSE) WDM BASED PONS}

Recent experiments of DSP-based PON's in real-time operation can be found in [13], and many optimization schemes have been also proposed with both single polarization (SP) and dual polarization (DP) approaches [14][15]. Compared to the SP, despite almost doubling the required analog hardware, the DP strategy with both encoded in phase and quadrature signals of each polarization doubles the spectral efficiency (SE) and therefore, for the same target data rate, it reduces the analog bandwidth and sampling rate requirements by half. In addition, it does not requires any solution for the polarization control in analog domain, since the polarization can be efficiently tracked using advanced DSP algorithms.

Figure 11(a) depicts an example of a DSP design for intradyne coherent detection combined with phase-based modulation formats and SP. The schematic includes pulse shaping and pre-equalization subsystems at the transmitter side for signal optimization, and normalization and clock, frequency and phase recovery algorithms at the receiver side. The normalization unit is used to improve the dynamic range of the DSP, ensuring that the amplitude of the digital signal spans over the entire excursion of the DSP bit-width resolution. The clock recovery is required to restore the sampling clock of the
ADCs, and the remaining subsystems are employed to recovery the signal carrier. i.e. frequency and phase. The standard method for carrier recovery is a phase-locked loop (PLL), which could eventually avoid the use of DSP for certain coherent network scenarios as coherent UDWDM-PON, however PLLs units are sensitive to the propagation delay inside the loop, leading to instability for high optical data throughput [15]. Therefore, DSP methods based on data-aided or nondata-aided algorithms have been the most widely used schemes for carrier frequency and phase recovery, and the wide range of the proposed techniques separate the frequency and phase compensation, since many phase tracking schemes only operate correctly for residual frequency offsets [14].

A similar configuration is depicted in Figure 11(b), but for DP signals. At the transmitter side, the bit streaming is divided into two signals for different polarizations, and the digital processing is performed separately. The adaptive equalizer at the receiver is traditionally a multiple-input multiple-output (MIMO) filter with the main purpose of restoring the signal SOP [18]. Since the timing-error of the ADCs is generally the same for both polarization signals, it can be shared between the clock recovery units of each polarization. And the same happens with the frequency offset in the frequency recovery subsystem, since the frequency offset affects both polarization signals equally. With this schematic, the number of DAC/ADCs is duplicated compared to the SP approach, however for the same target data rate, the sample rate, degree of parallelization (DOP) of the DSP and analog bandwidth requirements is halved. Thus, if the complexity added by the adaptive equalizer is not considered, the hardware requirements in the DSP maintains approximately the same for the same target bit rate. Further details of the reported DSP can be found in [14] and [16].

Coherent ultra-dense WDM (UDWDM) PON, combined with advanced DSP, has been discussed in academia for many years as an attractive solution to solve the fast-growing demand for bandwidth in the OANs [17]. The concept is to share the data traffic between different customers through a virtual pointto-point (P2P) WDM system using dedicated downstream (DS) and upstream (US) wavelength channels per customer with an interleaved grid allocation generally lower than $5 \mathrm{GHz}$, and supporting symmetric data rates beyond $1 \mathrm{~Gb} / \mathrm{s}$ per end user [23]. The transmission benefits from a network without coexistence issues of traffic observed for instance in TDM transmissions, e.g. burst mode operation for the US direction, and generally these systems can deliver higher data rates since each user takes advantage of all received bandwidth. Note that a PON solution based on TDM and direct detection cannot go far beyond an aggregated 10 Gbaud bandwidth due to the chromatic dispersion in the C-band. Several techniques are being considered to be deployed in the O-band where dispersion is low and therefore not limiting, however making the signal more prone to nonlinear effects when considering either higher power, bandwidth or wavelength division multiplexing. 


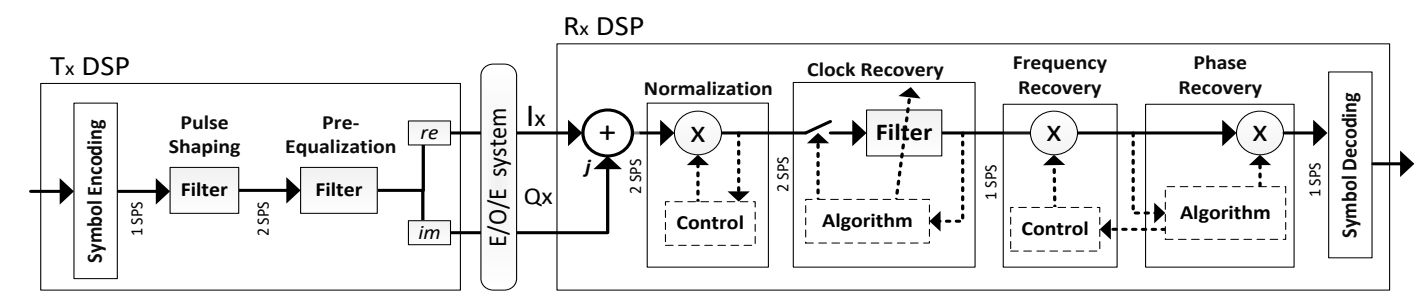

(a)

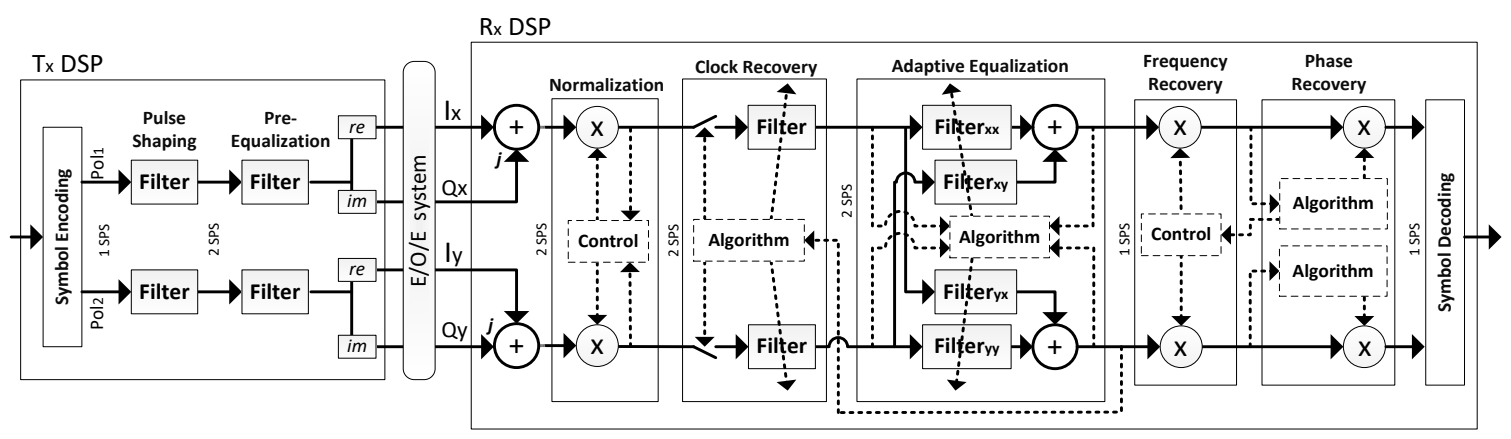

(b)

Figure 11 - DSP example for both OLT/ONU transmitters and receivers using intradyne coherent detection for (a) single polarization and (b) dual polarization signals (SPS: samples per symbol).

With coherent detection, advanced modulation formats using the amplitude, phase and polarization dimensions can also be employed for SE, and compared to a solution with direct detection, coherent enables higher sensitivity and wavelength selectivity, which allows an extended PON reach and an increased number of users. Combined with tunable optical sources, this solution provides high wavelength flexibility and a solution without any optical filter at the receiver side. In addition, by using coherent detection, efficient DSP algorithms for digital post-processing can be introduced to improve system performance or to increase the network flexibility by operating with different modulation formats or data rates. The DSP allows efficient shaping filtering, as Nyquist pulse shaping, which is a useful technique to achieve full usage of the available spectrum. This technique is a key ingredient for optimizing the number of users in an UDWDM grid, and the bidirectional concept can be efficiently implemented by pairing and interleaving DS and US bands, thus reducing linear and nonlinear crosstalk due to back-reflections or four-wave mixing (FWM) [23][17].

The schematic depicted in the Figure 12 shows an example of a bidirectional coherent PON using the virtual P2P UDWDM concept [17]. The salient feature of this network scheme is the wavelength plan of the DS and US channels, where a single optical carrier is signed for each ONU. For the DS direction, at the ONU the LO of the coherent receiver is tuned to the signed carrier. And after some electrical signal conditioning, which can be used to filter or to down convert the signal for baseband, the signal can be sampled by ADCs and recovered in DSP. For the US, the same tunable optical source as the LO is used for a single wavelength generation, and shifted by $\Delta \mathrm{F} / 2$ from the DS wavelength using electrical signal conditioning circuits, where $\Delta \mathrm{F}$ is the frequency channel spacing. At the OLT, an amplifier is a reasonable assumption as it can be used to boost the power of all received channels, and the channels can be detected using a single coherent receiver for various wavelengths, or various coherent receivers tuned for different wavelengths.

Although quite simple, this PON concept brings several challenges. The DS channels reach the ONU generally all with the same optical power, however the same may not be guaranteed for the US direction due to the different link budget between the OLT and each end-user, and thus it is necessary to ensure that the coherent receiver at the OLT has a good-enough dynamic power range. Nyquist pulse shaping brings also complexity and challenges for the hardware implementation (a) due to the required number of filter taps to generate an ideal shape, or also (b) due to its increase in the peak-to-average power ratio (PAPR) of the signal, requiring the use of higher effective number of bits (ENOB) in the DAC/ADCs and enhanced bit resolution in the DSP, or (c) due to the digital clock recovery algorithm at the receiver, since when the shaping roll-off factor tends to zero the clock information of the signal tends to be lost [16].

In addition, if any phase-based modulation format is also employed for bandwidth or receiver sensitivity optimization, the laser linewidth requirements must be also taken into account, otherwise the phase information of the signal after demodulation can be destroyed. The tolerable linewidth-tosymbol rate will depend on the performance of the DSP algorithm for frequency and phase tracking, which is another extra factor of complexity. The optical sources must also present a good-enough wavelength tunability control capable of operating steady for years, and resulting in signal frequency offsets much lower than $\Delta \mathrm{F} / 2$ in order to not affect the neighboring UDWDM channels. 


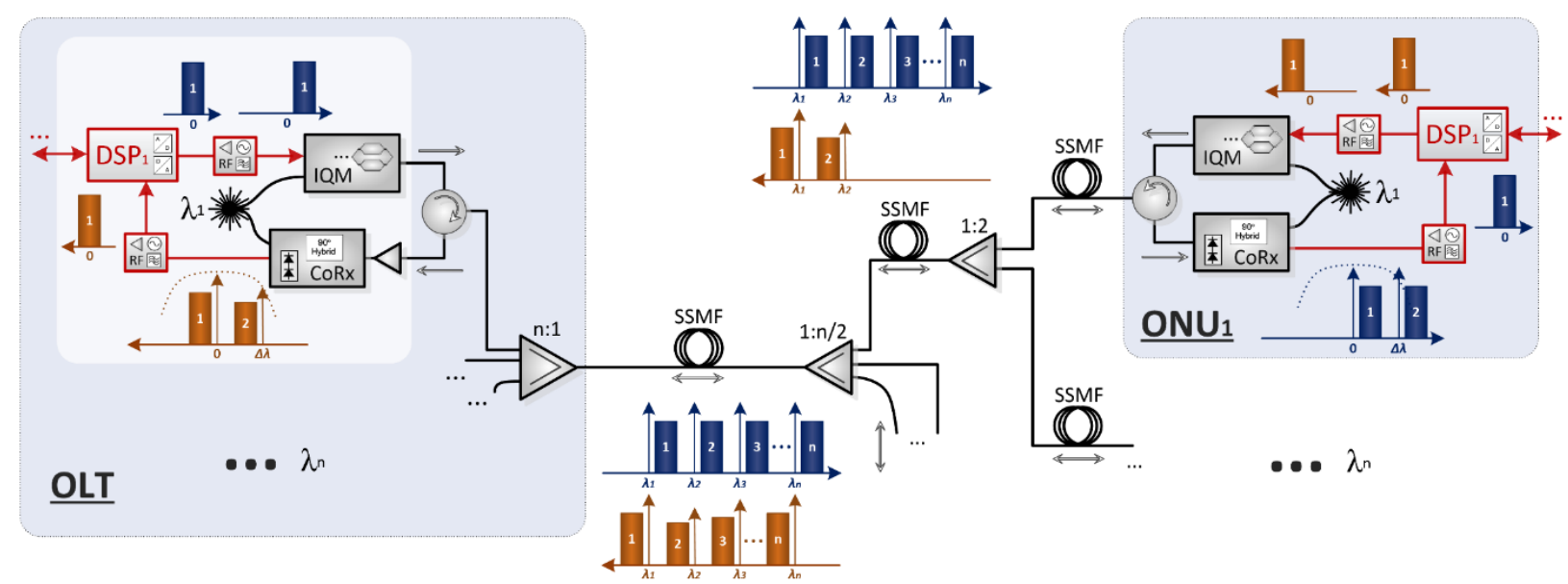

Figure 12 - UDWDM-PON system using a heterodyne coherent detection configuration

The UDWDM-PON can be an attractive solution if service providers can justify cost advantages or increase revenues offering a high capacity system. The introduction of the coherent solution in the access segment must be gradual to facilitate the use of legacy network infrastructure to the highest degree possible, assuring an economically network growth. Taking advantage of the high SE provided by advanced modulation formats, operating at low symbol rates, combining the recent advances in photonic integration, coherent PON may be a commercial reality in the future. The recent developments in high-speed electronic devices as application specific integrated circuits (ASICs) has been opening the possibility to implement advanced functionalities in digital domain, thus enabling to operate with advanced modulation formats and optical coherent solutions. The limited number of hardware resources becomes a major constraint for high-speed optical transmission systems. Thereby, low complexity DSP algorithms must be deployed in order to make possible the development of future coherent OANs.

DSP in PON has to be minimal, therefore complemented when possible with simplified analog functions which will enhance the performance and help removing complexity. When designing, the objective has to be reducing complexity to minimum and power dissipation in the same portion. For that, designing DSP for PON requires absolutely simplified algorithms, running on simplified physical layer, allowing the highest flexibility and performance.

\section{CONCLUSION}

In this work we have addressed most of the key types and features of the DSP based receiver related topics. The reception is always one of the system design constrains and mostly defines the most important parameters of the networks and its related standards. KK receivers will allow Direct and DMT with good quality. Coherent receivers, both in the simplified version and its more elaborated solutions will allow coherent multiwavelength filter-less transparent networks with high path loss tolerance and advanced formats when required. According to the choice DSP may or not be more or less complicated, however it seams clear that will always play a role both in the path compensation, or implementation of advanced functions. The operators and technology will dictate the next generations always subject to the main PON constrain which are cost and service evolution with as small ODN touch as possible.

\section{ACKNOWLEDGMENT}

D. Lavery is supported by the EPSRC under the TRANSNET grant (EP/R035342/1) and by the Royal Academy of Engineering.

A. Teixeira and R. Ferreira would like acknowledge the support of the project COMPRESS - PTDC/EEI-TEL/7163/2014; by FEDER, through the Regional Operational Program of Centre (CENTRO 2020) of the Portugal 2020 framework [Project HeatIT with Nr. 017942 (CENTRO-01-0247-FEDER-017942)], and the project Virtual Fiber Box with Nr. 033910 (POCI-01-0247-FEDER-033910). Additional support was provided by the COST action CA16220 European Network for High-Performance Integrated Microwave Photonics (EUIMWP) and IT (UID/EEA/50008/2013).

\section{REFERENCES}

[1] Full Service Access Network (FSAN). [Online]. Available: http://www.fsan.org/

[2] Y. Luo, X. Zhou, F. Effenberger, X. Yan, G. Peng, Y. Qian, and Y. $\mathrm{Ma}$, "Time-and wavelength-division multiplexed passive optical network (TWDM-PON) for next-generation PON stage 2 (NGPON2)". J. Lightw. Technol., vol. 31, no. 4, pp. 587-593 (2013).

[3] P. Chanclou, A. Cui, F. Geilhardt, H. Nakamura, and D. Nesset, "Network operator requirements for the next generation of optical access networks," IEEE Netw. Mag., vol. 26, no. 2, pp. 8-14, Mar./Apr. 2012

[4] J. Prat, et al., "Towards Ultra-Dense Wavelength-to-the-User: the Approach of the COCONUT project," Proceedings of ICTON 2013, paper Tu.C.3.2, IEEE, 2013.

[5] J. A. Altabas et al., "Real-Time 10 Gbps Polarization Independent Quasicoherent Receiver for NG-PON2 Access Networks," in Journal of Lightwave Technology, vol. 37, no. 2, pp. 651-656, 15 Jan. 15, 2019.

[6] M. Sezer Erkılınç, et al. "Comparison of Low Complexity Coherent Receivers for UDWDM-PONs ( $\lambda$-to-the-User)," J. Lightwave Technol. 36, 3453-3464 (2018)

[7] M. Presi, R. Corsini, M. Artiglia, and E. Ciaramella, "Ultra-dense WDM-PON $6.25 \mathrm{GHz}$ spaced $8 \times 1 \mathrm{~Gb} / \mathrm{s}$ based on a simplified coherent-detection scheme," Opt. Express 23, 22706-22713 (2015)

[8] Massimo Artiglia, et al., "Polarization-Independent Coherent RealTime Analog Receiver for PON Access Systems," J. Lightwave Technol. 34, 2027-2033 (2016) 
[9] I. N. Cano, A. Lerin,V. Polo, and J. Prat, "Polarization independent single-PD coherent ONU receiver with centralized scrambling in udWDMPONs,"in Proc. Eur. Conf. Opt. Commun., 2014, Paper P.7.12.

[10] J. Tabares, V. Polo, and J. Prat, "Polarization-independent heterodyne DPSK receiver based on $3 \times 3$ coupler for cost-effective udWDM-PON," in Proc. Opt. Fiber Commun. Conf., 2017, Paper Th1K.3

[11] A. Rafel and N. Parkin, "Cost analysis of coherent-based TRx PON network," in Proceedings of Eur. Conf. Opt. Commun. (ECOC), paper P7.16, Valencia, 2015.

[12] I. N. Cano et al., "Simplified polarization diversity heterodyne receiver for $1.25 \mathrm{~Gb} / \mathrm{s}$ cost-effective udWDM-PON," in Proc. Opt. Fibre Commun., 2014, p. W4G.2.B.

[13] R. M. Ferreira et al., "Real-Time Flexible Heterogeneous UDWDM System for Coherent PON," ECOC 2016, Düsseldorf Germany, paper W.4.P1.SC3.22.

[14] R. M. Ferreira et al., "Optimized carrier frequency and phase recovery based on blind Mth power schemes," PTL, vol. 28, no. 21, pp. 2439-2442, 2016.

[15] E. Ip and J. M. Kahn, "Feedforward carrier recovery for coherent optical communications," JLT, vol. 25, no. 9, pp. 2675-2692, September 2007.

[16] R. Ferreira et al., "Coherent UDWDM-PON with Dual-Polarization Transceivers in Real-Time,” PTL, vol. 29, no. 11, pp. 909-912, June 2017.

[17] A. Shahpari et al., "Coherent access: a review" JLT, DOI 10.1109/JLT.2016.2623793, 2016.

[18] S. J. Savory, "Digital filters for coherent optical receivers," Opt. Express vol. 16, no. 2, pp. 804-817 (2008).

[19] A. Mecozzi, C. Antonelli, and M.Shtaif, "KramersKronig coherent receiver,” Optica 3, 1220-1227 (2016).

[20] Z. Liu et al., "Practical Considerations on Discrete Multi-tone Transmission for Cost-effective Access Networks," in Proc. Optical Fiber Communication Conference, 2015, Paper M3J.4.

[21] E. Ciaramella, "Polarization-independent receivers for low-cost coherent OOK systems," IEEE Photon. Technol. Lett., vol. 26, no. 6, pp. 548551, Mar. 2014.

[22] D. Lavery, C. Behrens, and S. Savory, "A comparison of modulation formats for passive optical networks," Opt. Express vol. 19, no. 26, pp. B836-B841 (2011).

[23] J. D. Reis et al., "Terabit+ (192 10 Gb/s) Nyquist Shaped UDWDM Coherent PON With Upstream and Downstream Over a $12.8 \mathrm{~nm}$ Band," J. Lightwave Technol. vol. 32, 729-735 (2014).

[24] Z. Jia et al., "Digital Coherent Transmission for Next-Generation Cable Operators' Optical Access Networks," SCTE/ISBE Fall Technical Forum Cable-Tec Expo17, 2017.

[25] M. S. Erkılınc,, D. Lavery, K. Shi, B. C. Thomsen, R. I. Killey, S. J. Savory, and P. Bayvel, "Comparison of Low Complexity Coherent Receivers for UDWDM-PONs,” J. Lightwave Technol. vol. 36, no. 16, pp. 3453-3464, Aug. 2018.

[26] I. N. Cano, A. Lerin, V. Polo, and J. Prat, "Flexible D(Q)PSK 1.255 Gb/s UDWDM-PON with directly modulated DFBs and centralized polarization scrambling," in Proc. Eur. Conf. Opt. Commun., 2015, Paper Th.1.3.7.

[27] M. G. Taylor, "Phase Estimation Methods for Optical Coherent Detection Using Digital Signal Processing,” J. Lightwave Technol., vol. 27, no. 7, pp. 901-914, Apr. 2009.

[28] S. Kim, Takahiro Suzuki, Jun-Ichi Kani, and Akihiro Otaka, "Coherent Receiver DSP Implemented on a General-Purpose Server for a Full Software-Defined Access System," J. Opt. Commun. Netw. 11, A96-A102 (2019)

[29] T. Drenski and J. C. Rasmussen, "ADC \& DAC - Technology Trends and Steps to Overcome Current Limitations," in Proc. Optical Fiber Communication Conference, 2018, paper M2C.1

[30] C. Füllner, M. M. H. Adib, S. Wolf, J. N. Kemal, W. Freude, C. Koos, and S. Randel, "Complexity Analysis of the Kramers-Kronig Receiver,” Journal of Lightwave Technology, vol. 37 , no. 17 , (2019).

[31] C. Doerr, J. Heanue, L. Chen, R. Aroca, S. Azemati, G. Ali, G. McBrien, L. Chen, B. Guan, H. Zhang, X. Zhang, T. Nielsen, H. Mezghani, M. Mihnev, C. Yung, and M. Xu, "Silicon photonics coherent transceiver in a ball-grid array package," in Proc. Optical Fiber Communication Conference, 2017, paper Th5D.5.
[32] S. Gudyriev, C. Kress, H. Zwickel, J. N. Kemal, S. Lischke, L. Zimmermann, C. Koos, and J. C. Scheytt, "Coherent ePIC Receiver for 64 GBaud QPSK in0.25 $\mathrm{m}$ Photonic BiCMOS Technology,” J. Lightwave Technol., vol. 37, no. 1, pp. 103-109, Jan. 2019.

[33] Glance, "Polarization independent coherent optical receiver," J. Lightw.Technol., vol. LT-5, no. 2, pp. 274-276, Feb. 1987.

[34] L. G. Kazovsky et al., "ASK multiport optical homodyne receivers," Lightwave Technology, Journal of , vol.5, no.6, pp.770,791, Jun 1987

[35] M. Presi, F. Bottoni, R. Corsini, G. Cossu and E. Ciaramella, "All DFB-Based Coherent UDWDM PON With 6.25 GHz Spacing and a > $40 \mathrm{~dB}$ Power Budget," IEEE Photonics Technology Letters, vol. 26, no. 2, pp. 107-110, Jan.15, 2014.

[36] C. Xie, et al., "Colorless coherent receiver using $3 \times 3$ coupler hybrids and single-ended detection," Optics Express, vol. 20, no. 2 (2012).

[37] R.Noè, "Endless polarisation control in coherent optical communications," Electronics Letters , vol.22, no.15, pp.772,773, July 171986

[38] L. G. Kazovsky, "Phase- and polarization-diversity coherent optical techniques," Lightwave Technology, Journal of , vol.7, no.2, pp.279,292, Feb 1989

[39] Marco Presi, Massimo Artiglia, Fabio Bottoni, Mario Rannello, Ivan N. Cano, Jaison Tabares, Juan-Camilo Velásquez, Saeed Ghasemi, Victor Polo, Guang Yong Chu, Josep Prat, Gregorio Azcarate, Robert Pous, Chantal Vilá, Helene Debregeas, Gemma Vall-1losera, Albert Rafel, and Ernesto Ciaramella, "Field-Trial of a High-Budget, Filterless, $\lambda$-to-the-User, UDWDM-PON Enabled by an Innovative Class of Low-Cost Coherent Transceivers," J. Lightwave Technol. 35, 5250-5259 (2017)

[40] M. Rannello, M. Presi, and E. Ciaramella, "Optical vs. Electrical Duobinary Coding for $25 \mathrm{~Gb} / \mathrm{s}$ PONs based on DSP-free Coherent Envelope Detection," in Optical Fiber Communication Conference, OFC2018, paper M1B.6.

[41] W. Grover, "Error correction in dispersion-limited lightwave systems,” J. Lightw. Technol., vol. 6, pp. 643-654, May 1988.

[42] B. P. Smith, A. Farhood, A. Hunt, F. R. Kschischang, and J. Lodge, "Staircase codes: FEC for $100 \mathrm{~Gb} / \mathrm{s}$ OTN," J. Lightw. Technol., vol. 30, no. 1, pp. 110-117, Jan. 2012

[43] L. Schmalen, A. de Lind van Wijngaarden, and S. ten Brink, "Forward error correction in optical core and access networks," Bell Labs Tech. J., vol. 18, no. 3, pp. 39-66, Dec. 2013.

[44] IEEE LAN/MAN Standards Committee, "Draft standard for ethernet amendment: Physical layer specifications and management parameters for $25 \mathrm{~Gb} / \mathrm{s}, 50 \mathrm{~Gb} / \mathrm{s}$, and $100 \mathrm{~Gb} / \mathrm{s}$ passive optical networks," IEEE, Tech.Rep., Mar. 2018, IEEE P802.3ca/D1.0

[45] G. Coudlyzer et al., "A 50 Gbit/s PAM-4 Linear Burst-Mode Transimpedance Amplifier," IEEE Photon. Technol. Lett., vol. 31, no. 12, pp. 951-954, June 2019.

[46] R. Koma et al., "Burst-Mode Digital Signal Processing that PreCalculates FIR Filter Coefficients for Digital Coherent PON Upstream," J. Opt. Commun. Netw., vol. 10, no. 5, pp. 461-470, May 2018.

[47] S.-Y. Kim et al., "OLT Receiver for Power Normalization of Burst OFDM Signals Enabling OFDM/TDMA-PON," IEEE Photon. Technol. Lett., vol. 26, no. 24, pp. 2469-2472, Dec. 2014.

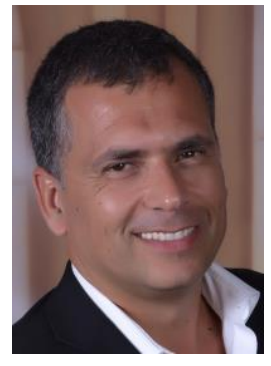

Antonio Teixeira, (M'96) got his $\mathrm{PhD}$ from university of Aveiro in 1999, partly developed at the University of Rochester. $\mathrm{He}$ holds an EC in management and leadership from MIT Sloan School and a post graduation in quality management in the field of Higher Education. He has been a professor at the University of Aveiro from 1999, being actually Associate professor with "Agregação". He has worked from 2009-13 in Nokia Siemens Networks and in Coriant (2013/14) as a standardization expert in the field of optical access (In FSAN, ITU-T, IEEE 802.3). From 2014, he is the Dean of the University of Aveiro Doctoral School aggregating $50 \mathrm{PhD}$ 
programs and 1300 students. He has published more than 400 papers (more than 130 in journals), has edited a book and contributed to several other. Holds 11 patents, and tutored successfully more than $70 \mathrm{MsC}$ 's and $14 \mathrm{PhD}$ 's, having participated in more than 35 projects (national, European and international). In 2014 he co-founded PICadvanced, a startup focused on providing solutions based on optical assemblies targeting biotech and optical networking (including access networks). He has served various conference committees, ECOC, OFC and others. Has been General Chair of ICTON 09, Networks 2014. He is a Senior Member of OSA and a member of IEEE and IEEE standards association.

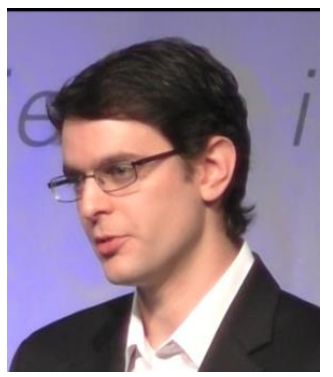

Domaniç Lavery received the MPhys degree in Theoretical Physics from Durham University, Durham, U.K. in 2009, and the Ph.D degree in Electronic and Electrical Engineering from University College London (UCL), London, U.K., in 2013.

He commenced a Royal Academy of Engineering Research Fellowship in December 2016 on the topic of "Simplified Transceiver Architectures for High Capacity Optical Networks", having previously worked as a Postdoctoral Research Associate on the EPSRC UNLOC project, investigating digital signal processing for optical fibre communications. He is a co-investigator in the EPSRC TRANSNET project; a multidisciplinary research programme investigating intelligent resource allocation in dynamic optical networks.

Dr. Lavery was a member of the Optical access networks (N4) subcommittee for OFC (2015-2017, chair 2019) and, additionally, has served on the technical programme committee for ECOC since 2018. He was awarded the IEEE Photonics Society Graduate Student Fellowship in 2012, and the Marconi Society's Paul Baran Young Scholar Award in 2013.

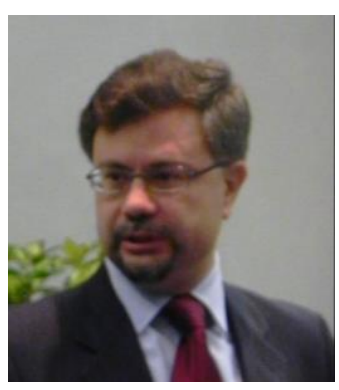

Ernesto Ciaramella is Full Professor of Telecommunications at Scuola Superiore Sant'Anna, Pisa, Italy. He previously worked at Alcatel, at Fondazione Ugo Bordoni (FUB), at the Centre for Studies and Laboratories in Telecommunications (CSELT) and at CNIT. His research interests include various areas of research in optical communications (components, systems, networks). His main research contributions are related to devices for the regeneration of the optical signals, design of WDM systems for transport and access, and free-space optical systems (optical wireless). He participated in several European research projects (OPEN, PHOTOS, ATLAS, NOBEL, SUNRISE, INFIERI). He coordinated the PRIN TOSCA and the EU-FP7 project COCONUT, on coherent systems for WDM PON access networks. He is now principal investigator of ESA- TOWS project. $\mathrm{He}$ is co-founder of Bright101. Prof. Ernesto Ciaramella served as a member of the Technical Programme Committee (TPC) of OFC and ECOC, was Lead Chair of
"Photonics in Switching 2012" and Co-Chair of the TPC of ACP 2012. He was organizer and co-chair of the first International Workshop on Optical Wireless (Pisa). He is author or co-author of about 200 publications and 24 international patents.

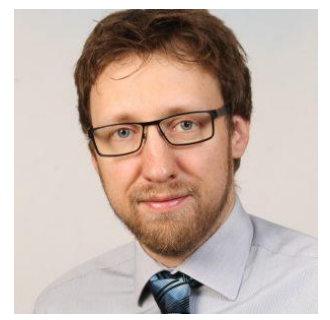

Laurent Schmalen received both his Dipl.-Ing. Degree and his Dr.-Ing. $(\mathrm{PhD})$ degree from RWTH Aachen University, Germany, in 2005 and 2011, respectively. Between 2011 and 2016, he was a research engineer in Alcatel-Lucent Bell Labs in Stuttgart, Germany, where he worked on forward error correction schemes for high speed optical communications. Between 2016 and 2019 he was a department head in Nokia Bell Labs in Stuttgart Germany. He served as guest lecturer at University of Stuttgart between 2014 and 2019 teaching a graduate-level course on modern error correction techniques. Since 2019, he is a full professor at Karlsruhe Institute of Technology (KIT) where he heads the communications engineering lab (CEL). Among others, he received the E-Plus award and the Friedrich-Wilhelm award for his $\mathrm{PhD}$ thesis, the Bell Labs president award and is a recipient of the best student paper award at the IEEE Signal Processing Systems (SiPS), the best paper award at the 2010 ITG conference on speech communications, and the 2016 Journal of Lightwave Technology best paper award. Additionally, he received the 2014 IEEE Transactions of Communications exemplary reviewer award and the 2018 Journal of Lightwave Technology Outstanding Reviewer recognition. He is a senior member of the IEEE.

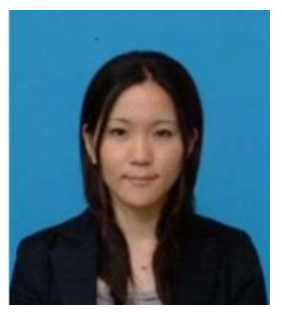

Noriko Iiyama received the B.E. degree in system design engineering and the M.E. degree in integrated design engineering from Keio University, Kanagawa, Japan, in 2006 and 2008, respectively.

Since 2008, she has been with the NTT Access Network Service Systems Laboratories, where she is engaged in the research of optical access networks and systems.

Ms. Iiyama is a member of the Institute of Electronics, Information, and Communication Engineers (IEICE), Japan. She received the Best Paper Award from the 18th Optoelectronics and Communications Conference (OECC) in 2013.

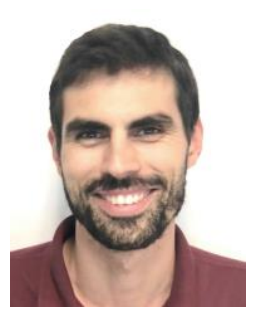

Ricardo M. Ferreira was born in Barcelos, Portugal, in 1989. He received m.sc. degree in electronic and telecommunications engineering from University of Aveiro, Portugal, in July 2012. Then he received his $\mathrm{Ph} . \mathrm{D}$. at the same university, and with Instituto de Telecomunicações from University of Aveiro, in December 2017, with a thesis work on digital signal processing (DSP) for flexible optical access networks based on coherent communications. He has published and contributed for various international journal and conference papers, as well as patents 
related to the optical communications, and he has been involved in several national and international projects. He joined the PICadvanced Company in December 2017 as a research engineer on optical systems, being his current work focused on DSP for high-speed transmission systems, as well as on the design of new transceivers for the next-generation optical access networks.

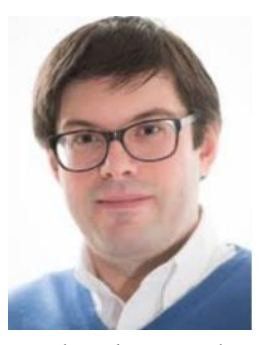

Sebastian Randel (S' 01-M' 06-SM' 13) received the Dr.-Ing. degree for his work on high-speed optical-time-divisionmultiplexed transmission systems from Technische Universitat Berlin, Berlin, Gremany, in 2005. Technology, where he is heading the Institute of Photonics and Quantum Electronics. From 2005 to 2010, he was a Research Scientist at Siemens Corporate Technology, Munich, Germany, where he led research and standardization activities in the fields of polymer- optical-fiber communication, visible-light communication, and optical access networks. From 2010 to 2016, he was a Member of Technical Staff at Bell Laboratories, Holmdel, NJ, USA. In this role, he designed, tested, and implemented power-efficient DSP algorithms for high-performance coherent optical transceivers. He first demonstrated that a digital multiple-input multiple-output equalizer can fully compensate mode coupling in few-mode fibers. In 2014, he demonstrated dual-polarization coherent-optical 64-QAM transmission at a record-high symbol rate of 72 Gbaud enabled by advanced DSP algorithms. He has (co-)authored more than 200 publications in peer-reviewed journals and conference proceedings and he filed more than 30 patent applications. His current research is focused on densely integrated optical communication systems.

Dr. Randel received the 2012 best paper award from the IEEE/OSA JOURNAL OF LIGHTWAVE TECHNOLOGY. He serves as a subcommittee chair of the Optical Fiber Communication Conference 2018. 